\title{
Role of MRI in Characterization of Occult Breast Lesions in High Risk Population
}

Caroline AY Habi., Hisham MA Mansour, Mohsen G Hassan, Ayman M IbrahimS.

Radiodiagnosis department, Faculty of Medicine, Ain Shams University

\begin{abstract}
OBJECTIVE: To evaluate the diagnostic validity of breast MRI in discriminating benign from malignant lesions in women with occult breast lesions who are at a high risk of developing breast cancer, with histopathologic findings and/or follow up used as the reference standard.
\end{abstract}

PATIENTS AND METHODS: Contrast-enhanced bilateral breast MRI was performed on 100 women at high risk of developing breast cancer with indeterminate imaging findings by mammography and/or ultrasonography. Lesions detected by MRI that could represent potential malignancies in both breasts were evaluated. Morphologic assessment and kinetic analysis (contrast enhancement and time/intensity curves) were performed on each lesion using dedicated postprocessing and display software. Functional MR tools were used in about $60 \%$ of cases to help in the differential diagnosis between malignant and benign of suspicious lesions detected at conventional MRI.

RESULTS: Among 100 patients included in the study $12 \%$ of the patients were finally diagnosed as free of any pathology, $56 \%$ had benign findings, while $31 \%$ of the patients were malignant. (24 IDC, 4 Invasive Lobular Carcinoma, 1 Medullary carcinoma, 1 Mucinous carcinoma and 1 DCIS.) The sensitivity, specificity, positive and negative predictive values of CE-MRI, were 100\%, 93\%, 86\%, and $100 \%$ respectively. Overall accuracy of MRI breast was 95\%. CONCLUSION: Breast MRI is highly effective in detection and characterization of occult breast lesions in high risk population, with excellent sensitivity and high specificity. Development of functional MRI tools contributed to the improving validity of this modality

Key words: MRI, Occult Breast Lesions

\section{INTRODUCTION}

Breast cancer is the most common malignancy that affects women worldwide and is a significant health care problem. Several well-established clinical imaging modalities have been in use to study the architecture, physiology and function of breast cancer. Methods such as X-ray mammography, ultrasound and physical examination are often limited in sensitivity and specificity, especially in young women. MRI is increasingly being used for preoperative local staging, localization of multiple lesions and screening of high-risk patients (Jagannathan, 2009). The standard breast MRI examination uses a gadolinium contrast agent to highlight lesion and their extent. In the assessment of breast cancer, measurements are usually acquired dynamically before, during and at certain time points after bolus injection of the contrast agent, allowing the contrast agent uptake and washout characteristics of the lesion to be characterized. Evaluation is based on the 
uptake curve from regions of interest defined within the enhancing parts of the lesion and on the morphology of the lesion (Leach, 2009). MR imaging of the breast is noninvasive and uses no ionizing radiation. Its primary benefit is high sensitivity, the highest of any imaging technique for breast lesions, high soft-tissue contrast, multiplanar sectional imaging and 3D rendering, the ability to detect small volume residual tumor and measurement of lesion size that corresponds with pathological measurement. Use of dynamic contrastenhanced MR helps to noninvasively image the microvascular network of tumors to determine if they are benign or malignant and to map functional parameters of breast lesions (Odle, 2006) The acquisition of 3D MRI data allows assessment of lesion morphology and contrast enhancement (tumor kinetics) regardless of breast size or architecture (Wiener et al, 2005) Morphology arises from the high spatial resolution afforded by MR imaging and deals with how the lesion looks, while kinetic features arise from temporal resolution or dynamic imaging and address how the lesion handles contrast uptake and washout (Odle, 2006).

Breast MRI has a very high sensitivity of greater than or equal to $90 \%$ for breast cancer. Studies of the use of breast MRI in high risk groups, have reported a specificity of 93 to 99\% (Lo and Cheung, 2008). Clinical indications for breast MRI include analysis of indeterminate breast lesions, screening of women with high genetic-familial risk, preoperative staging, evaluation of response to chemotherapy (Schmitz et al, 2008), evaluating the extent of disease in women diagnosed with breast cancer, identification of multicentric and multifocal disease (Friedman et al, 2008), differentiating between scar tissue and recurrent cancer, examining breasts that contain implants and diagnosis of metastatic breast cancer with unknown primary origin (Macura et al, 2006). An increased risk for breast cancer can be due to a personal history of breast cancer; a history of a breast biopsy, with "borderline" biologic behaviour such as radial scar, lobular carcinoma in situ, or atypical ductal hyperplasia; a history of mediastinal irradiation for Hodgkin disease; a familial history of breast and/or ovarian cancer; or genetic mutations. For all of these subgroups, breast MR imaging has been successfully used to help detect mammographically and sonographically occult breast cancer (Kuhl, 2007). In the current study we investigated the diagnostic accuracy of breast MRI in discriminating benign from malignant lesions in women with occult breast lesions who are at a high risk of developing breast cancer, with histopathologic findings or follow up used as the reference standard for comparison.

\section{PATIENTS AND METHODS}

This study included 100 female patients referred to perform Contrast-enhanced bilateral breast MRI at Ain Shams University hospitals and private radiological centres. All patients were at high risk of developing breast cancer with normal or indeterminate imaging findings by mammography and/or ultrasonography.

\section{Exclusion criteria included:}


The following patients were excluded from the current study:

1. Patients with bad general condition and renal impairment.

2. Lactating and pregnant females.

3. Patients known to have contraindications for MRI e.g. an implanted magnetic device, pacemakers, claustrophobia.

All patients were subjected to complete clinical and radiological evaluation assessment. This involved thorough history taking including personal history, especially with respect to previous breast cancer or biopsies with benign histology, family history of breast or ovarian cancer, abnormalities suspicious of malignancy (e.g., palpable mass, skin retraction, nipple discharge), hormonal status and previous allergic reaction after administration of MR contrast material. Previous imaging studies such as mammography and/or sonography, and their findings were evaluated and recorded. Then contrast-enhanced bilateral breast MRI was performed and results of MRI examination were compared to the findings from histopsthology and/or follow up.

Patient preparation: There is no specific preparation for different MR imaging such as fasting. When possible, MRI examination of the breast in premenopausal women was scheduled to be performed in the second or third week of the cycle, unless urgent. For application of the contrast material, a venous line (18-20 G) was inserted before starting the examination. Every patient was screened for ferromagnetic objects, implanted medical devices, surgical clips, metal fragments in or near the eyes, and other metallic objects. All metallic objects were removed from the patient's body

Scan protocol: MR imaging was performed with Philips superconductive magnet system operating at 1.5 Tesla using breast surface coils. The patient lies prone on the examination couch with her breast(s) positioned dependent in the receiver breast coil(s) and the arms placed along the body. Appropriate IV anesthetic agents were given to some patients who feared the MRI machine when needed. IV contrast (gadolinium chelates) was given for assessment of tumor kinetics. Dose given was about $0.2 \mathrm{ml} / \mathrm{kg}$ body weight. Spine-echo T1 WI was performed after contrast administration. The routine protocol applied in this study included Axial T1, T2, Axial T2 fat suppressed, STIR or SPAIR \pm Sagittal STIR, Axial Post-contrast T1 WI fat suppressed \pm Sagittal Post-contrast 3D TFE (T1 WI). Dynamic 3D multiphase post-contrast study was done in 6-8 minutes with MIP reconstruction (once before contrast and 4-5 times after contrast, each around 1 minute). For any region of interest (ROI), Time-Signal intensity curves were performed. Signal intensity measurements were performed prior to as well as following contrast administration in this region of interest (ROI). ROIs are drawn at the point of maximum enhancement. Diffusion weighted imaging + ADC calculation were utilized in 59 cases. The field of view (FoV) typically ranged from 280 to $340 \mathrm{~mm}$, depending on the breast size. The slice thickness was $3 \mathrm{~mm}$ or sometimes $2 \mathrm{~mm}$, and without gaps. Perfusion imaging was 
performed in 2 cases and MR Spectroscopic imaging was performed in 10 cases.

Table (1) Physical parameters of different pulse sequences.

\begin{tabular}{|l|l|l|l|l|}
\hline & Axial T1WI & Axial T2WI & Axial/ Sagittal STIR & $\begin{array}{l}\text { Axial/ Sagittal }+\boldsymbol{c} \\
\text { T1WI Fat sat }\end{array}$ \\
\hline TR & 540 & $4000-4800$ & $2000-7500$ & 485 \\
\hline TE & 10 & 120 & $55-170$ & 10 \\
\hline NEX & 1 & 1 & 1 & 1 \\
\hline ST & $3 \mathrm{~mm}$ & $3 \mathrm{~mm}$ & $3 \mathrm{~mm}$ & $3 \mathrm{~mm}$ \\
\hline Gap & 0 & 0 & 0 & 0 \\
\hline FOV & $34 \times 34 \mathrm{~cm}$ & $34 \times 34 \mathrm{~cm}$ & $34 \times 34 \mathrm{~cm}$ & $34 \times 34 \mathrm{~cm}$ \\
\hline Matrix & $\begin{array}{l}256 \times 160 \\
\text { or } 256 \times 192\end{array}$ & $\begin{array}{l}256 \times 160 \\
\text { or } 256 \times 192\end{array}$ & $\begin{array}{l}256 \times 160 \\
\text { or } 256 \times 192\end{array}$ & $\begin{array}{l}256 \times 160 \\
\text { or } 256 \times 192\end{array}$ \\
\hline
\end{tabular}

TR: Repetition time TE: Echo time NEX: Number of acquisition FOV: Field of view STIR: Short time inversion recovery Fat sat: Fat saturation ST : Slice thickness.

\section{Data interpretation:}

All lesions or areas of abnormal enhancement detected by MRI that could represent potential malignancies in both breasts were evaluated, by experienced MR radiologist, as regard:

- Morphology

- Exact Location

- Extent of involvement

- Signal intensity on different pulse sequences

- Kinetics; Enhancement pattern and time/intensity curves

- Vascularity of the lesion

ACR BI-RADS-MRI Lexicon was used as a guideline for data collection. According to the BI-RADS Lexicon of the American College of Radiology, suspicious enhancing areas in the breast are differentiated into (a) focus/foci, (b) masses, or (c) areas of nonmass-like enhancement. Moreover, associated findings are described (Fischer, 2010). Masses and areas of non-mass like enhancement are subjected to careful analysis of their morphology, enhancement kinetics, and signal intensity patterns on $\mathrm{T} 1$ - and $\mathrm{T} 2$-weighted images (Lehman et al, 2005 and Kuhl, 2007). A focus is a small isolated spot of enhancement, generally less than $5 \mathrm{~mm}$ in size, that is so tiny that no definitive morphologic descriptors can be applied (Fischer, 2010, Lehman et al, 2005 and Kuhl, 2007). A mass is a three-dimensional space-occupying lesion that may or may not displace or otherwise affect the surrounding normal tissue. For the evaluation of masses, different criteria are described. Criteria include shape, margin, endotumoral type of contrast internal enhancement, and the initial and post-initial signal behavior in relation to the precontrast signal. Non-mass-like lesions on MRI of the breast are enhancing areas that are neither a 
focus nor a mass. Non-mass-like enhancement descriptions included distribution, internal enhancement and symmetry. It is described as a focal area, linear, ductal, segmental, regional, multiple regions, or diffuse. Internal characteristics of the enhancing area, like homogeneous, heterogeneous,

stippled/punctuate, clumped,

or reticular/dendritic, is evaluated (Fischer, 2010 and Lehman et al, 2005). Associated findings (such as edema, adenopathy, cysts, and skin or chest wall involvement) are reported and kinetic curve assessment of all lesions described. The analysis of enhancement kinetics included initial peak (Early phase) enhancement and delayed-phase enhancement analyses, by measuring the signal intensity in region of interest (ROI), and tracking its course over the dynamic series (time-signal intensity curve). ROIs were placed into the area that exhibits strongest enhancement on the first postcontrast image. Early Postcontrast Phase enhancement describes the steepness of the first part of the kinetic curve, indicating the velocity and degree with which enhancement occurs and may be slow, medium, or rapid. Delayed phase enhancement refers to signal intensity changes that occur immediately after the early signal intensity increase which may (a) decline again; (b) exhibit a sharp bend and plateau; or (c) continue to rise after the early phase, yielding persistent enhancement. Enhancing nodules were assumed to be almost malignant when they showed early intense enhancement and progressive signal loss over time (washout), whereas lesions showing progressive enhancement over time were assigned to be more likely benign (Kuhl, 2005). (Fig. 1)

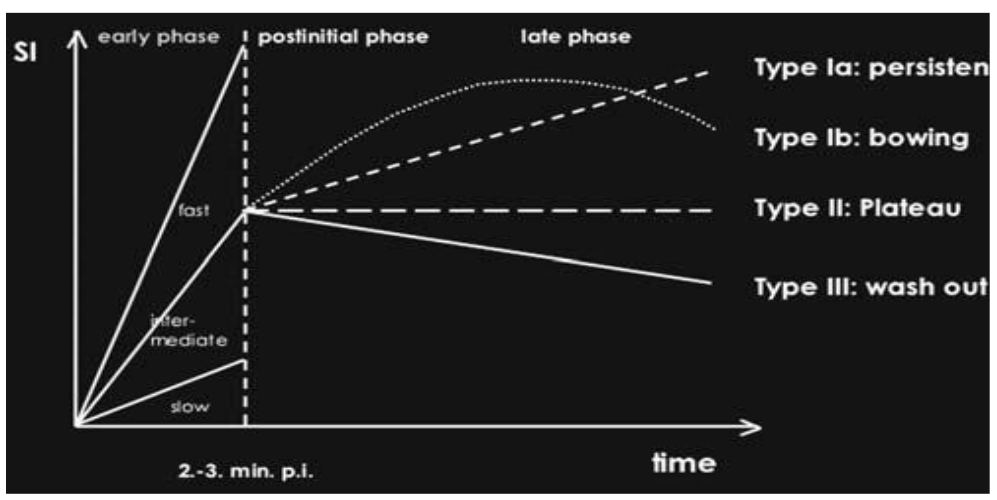

Fig. 1 Different phases of the signal time course of a dynamic series and the respective kinetic criteria. We distinguish three phases: (1) initial post-contrast phase (injection of contrast agent until the second post-contrast minute), (2) post-initial phase (second through third post-contrast minute), (3) late post-contrast phase (4th through 8th poscontrast minute). In the postinitial and late postcontrast phase, the time course of signal intensity is rated visually according to the following scheme: Type Ia ("persistent enhancement"). Type Ib ("persistent with bowing"). Type II ("plateau curve"). Type III ("washout curve") (Kuhl, 2005).

\section{Using ACR BI-RADS-MRI Lexicon,} lesions were categorized into seven categories according to the findings of the breast MRI. MRM-BI-RADS 0 describe an incomplete assessment and the category MRM-BIRADS 6 is given to a histological verified breast carcinoma. The other five categories include: Category MRM-BI-RADS 1: "negative" No abnormal enhancement is found. Category 
MRM-BI-RADS 2: "benign" MRI shows a benign finding, for example a hyalinized nonenhancing fibroadenoma, cysts, and old nonenhancing scars, fat-containing lesions such as oil cysts, lipomas, galactoceles, or mixed-density hamartomas. Category MRMBI-RADS 3: "probably benign" Changes that are highly unlikely to be malignant, i.e., those that have a very high probability of being benign, are placed in this category. Category MRM-BI-RADS 4: "suspicious" These are lesions that do not have the characteristic morphology of breast carcinoma, but do have a definite low to moderate probability of being malignant. Category MRM-BI-RADS 5: "highly suggestive of malignancy" Lesions categorized as MRM-BI-RADS 5 have a high probability of being cancerous. They show the typical findings of a malignant breast tumor (Fischer, 2010).

\section{Results}

A total of 100 high risk females presented with occult breast lesions were included in the study. Cases included in the study ranged from 22 to 67 years old with mean age of 43.7 years. $38.7 \%$ of the malignant cases were aged from 41 to 50 years, $25.8 \%$ from 31 to 40 years old and $35.5 \%$ were $\geq 51$.

Clinical data: Palpable breast masses was the most common clinical presentation (31 patients, 31\%) among the study population, 28 patients (28\%) were asymptomatic, 29 patients (29\%) were referred for follow up postsurgery, 3 patients (3\%) had skin changes, 3 patients $(3 \%)$ complained of breast enlargement, 3 patients (3\%) had enlarged axillary lymph nodes, 2 patients (2\%) had nipple discharge and only one patient (1\%) complained of breast pain. Positive family history of breast cancer was the most common risk factor encountered in the studied group $(38 \%)$. Other risk factors were; history of previous breast cancer (29\%), personal history of another malignancy such as endometrial or ovarian cancer (6\%), biopsy-proven diagnosis of proliferative breast disease, atypia, lobular carcinoma in situ or radial scar (9\%), increased breast densities in mammography relative to age $(11 \%)$ and late first term pregnancy or nulliparity $(7 \%)$.

\section{Histopathology findings and follow}

up: Among the 100 patients with occult breast lesions included in this study 31 patients (31\%) turned out to be malignant, 2 patients had multicenteric malignant lesions and 4 patients had 2 malignant lesions either epsilateral or contralateral. 56\% (56 patients) of the studied lesions were benign; 52 cases had various benign pathologies, (hyperplasia, fibrocystic disease, fibroadenoma, benign postoperative changes), 3 cases had benign Phylloids tumour and 1 case was Intraductal pappiloma. 13 cases (13\%) were diagnosed as free of pathology. The histopathalogical types of the 35 malignant biopsies (taken from 31 patients) in our study were as following: 24 cases IDC (77.4\%), 4 cases invasive lobular carcinoma (13\%), one case medullary carcinoma $(3.2 \%)$, one case mucinous carcinoma (3.2\%) and one case DCIS (3.2\%). The commonest location of the malignant masses within the breast tissue was in the 
upper outer quadrant, where $51.4 \%$ of the lesions were located.

Mammographic results: The results of the mammographic examinations of the patients were; $38 \%$ of the studied cases had dense breasts in mammography which hindered proper assessment of the breast masses, $7 \%$ showed normal mammography with no masses detected, $31 \%$ were categorized as BIRADS III (probably benign), $3 \%$ were categorized as BIRADS IV and V (probably malignant and highly suspicious of malignancy), $21 \%$ were described as having irregular densities for further assessment. Among the 38 cases that had dense breasts, 12 cases turned out to have underlying malignant masses, 9 cases had underlying benign pathologies and the rest (17 cases) were free. All these lesions were readily identified in MRI. The sensitivity, specificity, positive and negative predictive values of mammography were found to be $51.6 \%, 88.4 \%, 66.7 \%$, and $80.3 \%$ respectively. Overall accuracy of mammography was $77 \%$.

Table (2) Distribution of the studied group as regards the mammographic findings

\begin{tabular}{|l|l|l|}
\hline Variables & No & $\%$ \\
\hline NAD & 7 & $7 \%$ \\
\hline Dense breast (non conclusive) & 38 & $38 \%$ \\
\hline Probably Benign (BIRADIII) & 31 & $31 \%$ \\
\hline Probably malignant (BIRAD IV \& V) & 3 & $3 \%$ \\
\hline Irregular asymmetric densities & 21 & $21 \%$ \\
\hline Total & 100 & $100 \%$ \\
\hline
\end{tabular}

Table (3) Comparison between mammography versus histopathology and follow up among the studied group

\begin{tabular}{|l|l|l|l|}
\hline Mammography & \multicolumn{2}{|l|}{$\begin{array}{l}\text { Pathology } \\
\text { Benign }\end{array}$} & Palignant \\
\hline $\begin{array}{l}\text { Probably Benign and } \\
\text { Dense breast }\end{array}$ & $61(88.4 \%)$ & $15(48.4 \%)$ & $\begin{array}{l}>0.05 \\
\text { NS }\end{array}$ \\
\hline Probably Malignant & $8(11.6 \%)$ & $16(51.6 \%)$ & \\
\hline
\end{tabular}

This table shows no statistically significant association between mammography and pathology by using chisquare test. $\mathbf{P}$ value $>0.05$ insignificant

Table (4) Validity of mammography in case of breast lesions among the studied cases:

\begin{tabular}{|l|l|}
\hline Variables & $\mathbf{\%}$ \\
\hline Sensitivity & $51.6 \%$ \\
\hline Specificity & $88.4 \%$ \\
\hline PPV & $66.7 \%$ \\
\hline NPV & $80.3 \%$ \\
\hline Accuracy & $77 \%$ \\
\hline
\end{tabular}


MRI results: The results of the MRI examinations of the patients were; $64 \%$ of the studied cases were benign, BIRADII and BIRADIII, by MRI criteria and $36 \%$ of cases were suggestive of malignancy by MRI criteria (BIRADIV and BIRADV). Among the 36 cases diagnosed by MRI as malignant, 5 turned out to be benign by histopathological evaluation (false positive), while among the 64 cases diagnosed by MRI to be benign lesions, none were proved to be malignant by histopathological evaluation (no false negative) The sensitivity, specificity, positive and negative predictive values of MRI for occult breast lesions in high risk patients included in the study, were found to be $100 \%$, $93 \%, 86 \%$, and $100 \%$ respectively. Overall accuracy of MRI breast was $95 \%$.

Table (5) Distribution of the studied group as regard MRI findings

\begin{tabular}{|l|l|l|}
\hline Variables & No & $\%$ \\
\hline Benign (BIRADII) & 45 & $45 \%$ \\
\hline Probably Benign (BIRADIII) & 19 & $19 \%$ \\
\hline BIRADIV & & \\
A & 9 & $9 \%$ \\
B & 7 & $7 \%$ \\
C & 11 & $11 \%$ \\
\hline BIRADV & 9 & $9 \%$ \\
\hline
\end{tabular}

Table (6) Comparison between MRI versus histopathology and follow up among the studied group

\begin{tabular}{|c|c|c|c|c|}
\hline MRI & $\begin{array}{l}\text { Patholog. } \\
\text { Benign }\end{array}$ & alignant & $\mathrm{X}^{2}$ & $\mathbf{P}$ \\
\hline Benign & $64(93 \%)$ & $\mathbf{0}$ & 47 & $\begin{array}{l}<0.001 \\
\mathrm{~S}\end{array}$ \\
\hline Malignant & $5(7 \%)$ & $31(100 \%)$ & & \\
\hline
\end{tabular}

This table shows statistically significant association between MRI and pathology by using chi-square test. $\mathrm{P}$ value $<0.01$ significant

Table (7) Validity of MRI in characterization of breast lesions among the studied cases

\begin{tabular}{|l|l|}
\hline Variables & $\%$ \\
\hline Sensitivity & $100 \%$ \\
\hline Specificity & $93 \%$ \\
\hline PPV & $86 \%$ \\
\hline NPV & $100 \%$ \\
\hline Accuracy & $95 \%$ \\
\hline
\end{tabular}


This table shows that MRI is considered better positive predictor for cancer breast in relation to the pathology with higher sensitivity than specificity but general speaking it is considered highly valid with high specificity also.

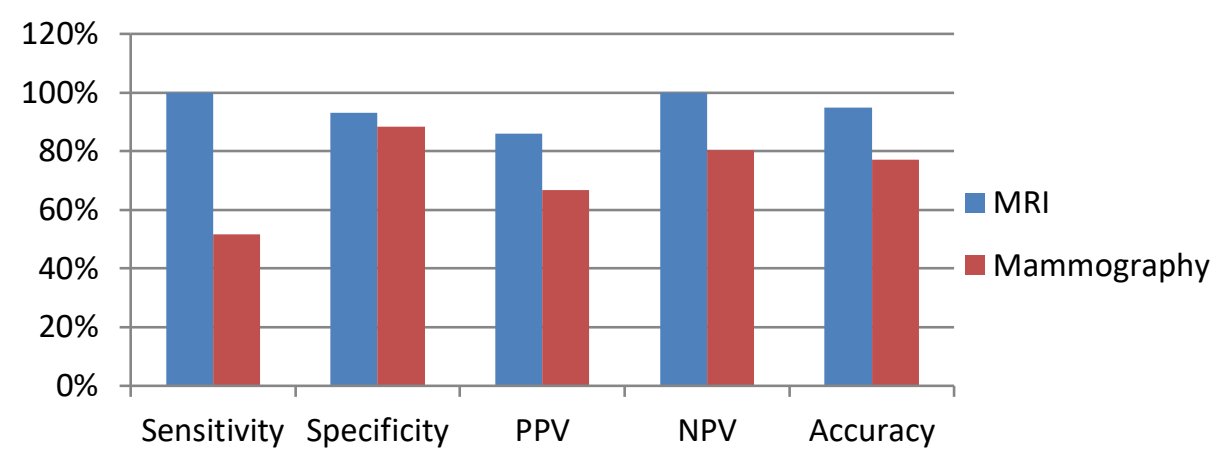

Fig. 2.: A column chart for comparison between validity of mammography versus MRI

\section{DISCUSSION}

Detection of breast cancer is the primary aim of breast imaging. The combination of decreased mammographic sensitivity and increased prevalence of breast cancer in denser breasts has prompted interest in the investigation of supplemental screening with ultrasound or MRI (Shafqat et al, 2011). Although increasing age is the single most important risk factor for developing breast cancer (Bevers, 2008), yet the rate at which risk rises declines significantly around age 50 years. Therefore breast cancer incidence in high risk population is higher in relatively younger age groups than in general population (Spicer and Pike, 2005). This was reflected among our study population who were all chosen to be of high risk of breast cancer, and so around $65 \%$ of cancer cases were presented in the $31-50$ years age group $(38.7 \%$ of the malignant cases were presented in the 41-50 years age group and $64.5 \%$ presented in the 31- 50 age group).

Until recently, the use of breast MR imaging for screening was greatly discouraged. This has changed, in that MR is increasingly used for screening in selected subsets of women with an increased lifetime risk for breast cancer (Kuhl, 2007). We decided to choose the patients in this study among the high risk population, because many studies proved that MRI is a highly sensitive test for detecting breast cancer, however it is not recommended for screening women at average risk. So it has been proposed as adjunct screening for women with a high risk, such as genetic predisposition, to breast cancer. Mammography has lower sensitivity in this group, detecting fewer than half the breast cancers diagnosed (Houssami et al, 2009). A meta-analysis of five studies of MRI as an adjunct to conventional imaging in high-risk women has provided convincing evidence that MRI detects additional cancers, compared with mammography alone (Houssami et al, 2009 and Lord et al, 2007). MRI is more sensitive than mammography in screening women with suspected or proven inherited mutations of the breast cancer genes. The addition of MRI in screening this population detects 8-24 
additional cancers per 1000 screens, but also increases a woman's risk of being recalled for investigation or surgical biopsy for falsepositive findings (Leach et al, 2005, Warner et al, 2004 and Sardanelli et al, 2007)

Subgroups in the population with an elevated risk of breast cancer can be identified by performing genetic testing for breast cancer predisposition mutations or by evaluating family history (Leach, 2009). High-risk populations include women with known or suspected gene mutations, women with firstdegree relatives who are known mutation carriers, and those with a family history suggesting inherited mutations (Houssami et $a l, 2009)$. An increased risk for breast cancer can also be due to a personal history of breast cancer; a history of a breast biopsy, with "borderline" biologic behavior such as radial scar, lobular carcinoma in situ, or atypical ductal hyperplasia; a history of mediastinal irradiation for Hodgkin disease; a familial clustering of breast and/or ovarian cancer; or dense breast composition. For all of these subgroups, breast MR imaging has been successfully used to help detect mammographically and sonographically occult breast cancer (Friedman et al, 2008 and Kuhl, 2007). In the current study we identified high risk patients by evaluating the personal and family history. Positive family history of breast cancer was the most common risk factor encountered in the studied group (38\%).

Bleiweiss et al (2005) stated that the two main histologic types of invasive carcinoma of the breast are invasive duct carcinoma and invasive lobular carcinoma. Together they constitute the vast majority of infiltrative malignancies that will be encountered in routine practice. Van de Vijver (1999) mentioned the estimated frequency of each histologic type of invasive breast cancer; Invasive Ductal carcinoma (not otherwise specified) $70 \%$, Invasive Lobular carcinoma 10\%, Tubular carcinoma 5\%, Mucinous carcinoma 5\%, Medullary carcinoma 3\%, Atypical Medullary carcinoma 3\% and others $4 \%$. In general this coincides with the results of our study; where IDC was the most frequent histopathological type encountered in the study malignant cases. (24 cases IDC (77.4\%), 4 cases invasive lobular carcinoma (13\%).

Of all breast imaging techniques that are currently available, including mammography, breast US, positron emission tomography, and scintimammography, MR offers the highest sensitivity for invasive breast cancer. Published sensitivity levels range between $89 \%$ and $100 \%$. In all studies that can be found in the literature, the sensitivity of MR imaging was higher than that of mammography. The degree to which the sensitivities of mammography and breast MR imaging differ in the same patients depend on the mammographic breast density and the type of breast cancer: The difference increases with increasing breast density and for cancers that are difficult to diagnose on the basis of mammographic findings. The sensitivity of breast MR imaging is not impaired by the amount or density of the fibroglandular tissue nor by scar tissue, radiation therapy, or prosthetic breast implants or other types of breast reconstruction (Kuhl, 2007). 
Overlooking invasive breast cancer on MR images is rare, but it certainly does happen. Non-enhancing invasive breast cancers are exceedingly rare. More often, the reason for failure to diagnose invasive cancer with breast MR imaging is early and strong enhancement in the surrounding normal fibroglandular tissue that may mask the enhancing cancer (Kuhl, 2007).

In the current study, among the 38 cases that had dense breasts by mammography, 12 cases turned out to have underlying malignant masses, 9 cases had underlying benign pathologies and the rest (17 cases) were free. All these lesions were readily identified in MRI. Therefore our results agree with Morrow et al (2011) who stated that, compared with mammography, MRI has a higher sensitivity for the detection of breast cancer and is not affected by breast density. Also these findings agree with previous studies that have cumulatively evaluated breast MRI in more than 1000 high-risk patients and found that the technique identified cancer that was not seen on mammography in 4 percent of cases

\section{(Liberman, 2004).}

In our study population the sensitivity, specificity, positive and negative predictive values of mammography were found to be $51.6 \%, 88.4 \%, 66.7 \%$, and $80.3 \%$ respectively. Overall accuracy of mammography was $77 \%$. $\mathrm{X}$-ray mammography (XRM) is an effective screening method in the normal population, particularly in those over 50, showing a sensitivity of about $86 \%$. However, with the early onset of disease in the high-risk population, there is a need to screen at a younger age, where the higher proportion of breast parenchyma can result in dense mammograms that are hard to interpret. Several studies (Kerlikowske et al, 1996 and Mushlin et al, 1998) have shown that, in the normal population, the sensitivity of screening mammograms falls with age (Leach, 2009). This explains the lower sensitivity achieved by mammography in this study high risk population. (51.6\%)

Most of the previous studies showed that the sensitivity of MR imaging for detection of breast cancer is very high, and approaches $100 \%$ for invasive carcinoma. However the specificity is lower and varies widely between different studies. The factors associated with this wide range of specificity are differences in the study population, strength of magnet, imaging protocols, and interpretation criteria (Bedrosian et al, 2003). In their study, Baltzer et al (2010) stated that false positive findings occur and lead to unnecessary biopsy and concluded that nonmass lesions were the major cause of falsepositive breast MRI findings. Therefore the basic drawback of this modality was low specificity for breast malignancy. Multiple studies, including the current study, have shown that with the improvement in equipment and technique there is gradual increase in specificity (Shafqat et al, 2011).

A number of prospective single- or multicenter trials have been conducted to evaluate the role of MR imaging in screening high risk population. The results of all these studies are concordant in that early diagnosis of cancer is substantially improved if MR is 
included in the surveillance program. Cancers were diagnosed at a prognostically favorable stage, with published sensitivity levels ranging between $79 \%$ and $98 \%$. As a result, MR imaging is now accepted as an integral part of surveillance programs in women with suspicion of familial breast cancer (Kuhl, 2007). The data regarding the specificity and positive predictive value for screening MR are less concordant: A higher rate of false-positive diagnoses for MR imaging than for mammography has been reported in several studies (Leach et al, 2005, Warner et al, 2004 and Kriege et al, 2004). The study by Warner et al (2004) provides a possible explanation for this: Whereas the rate of false positive MR diagnoses was high at the beginning of the breast MR screening project, the rate decreased from year to year to reach the same level as that for mammography, where mammography and MR exhibited equivalent positive predictive values. This observation, as well as the results from other studies (Kuhl,

2005, Stoutjesdijk et al, 2001 and Trecate et $a l, 2003)$ suggests that a high rate of falsepositive diagnoses is not inherent to the technique of breast MR imaging. Rather, it is due to limited experience with breast MR in a screening setting (Kuhl, 2007).

In our study the sensitivity, specificity, positive and negative predictive values of MRI for occult breast lesions in high risk patients included in the study, were found to be $100 \%$, $93 \%, 86 \%$, and $100 \%$ respectively. Overall accuracy of MRI breast was 95\%. Among the 36 cases diagnosed by MRI as malignant, 5 turned out to be benign by histopathological evaluation (false positive), while among the 64 cases diagnosed by MRI to be benign lesions, none were proved to be malignant by histopathological evaluation (no false negative). Therefore breast MRI had higher sensitivity than specificity but general speaking it is considered highly valid with high specificity also. We found that combining qualitative assessment of morphological appearance of lesion on post contrast study and time signal intensity curves with functional MR tools, which were utilized in about $60 \%$ of the study population, was useful for achieving high validity for breast MRI.

Our results are comparable to most of studies done in the past; Shafqat et al (2011) performed a retrospective analysis on 70 patients who underwent MRI breast because of suspicious mammographic abnormalities, yet not essentially high risk patients. The sensitivity, specificity, positive and negative predictive values of MRI for breast lesions was found to be $94 \%, 85 \%, 90 \%$, and $82 \%$ respectively. Overall accuracy of MRI breast was $90 \%$. There have been a number of studies that have investigated MRI and Mammography for screening women at high familial risk of breast cancer. A study performed by Kuhl et al (2000) included 192 patients with 12 detected cancers. They found that the sensitivity of breast MRI was $100 \%$ versus $33 \%$ for mammography, while MRI specificity was $95 \%$ versus $93 \%$ for mammography. Results of Podo et al (2002) are much similar; sensitivity and specificity were $100 \%$ and $99 \%$ for MRI respectively and $13 \%$ and $100 \%$ for Mammography. Tilanus- 
Linthorst et al (2000) conducted another study on 109 patients. They detected 3 cancer cases that were all seen on MRI. MRI sensitivity and specificity were $100 \%$ and $94 \%$ respectively. One year later Stoutjesdijk and colleagues (2001) performed a study on a population of 179 women with high risk of breast cancer. 14 cancers were detected with sensitivity and specificity $100 \%$ and $93 \%$ respectively for MRI and $42 \%$ and $96 \%$ for mammography. Another study performed by Warner et al (2001), achieved lower values as regard the validity of MRI; sensitivity and specificity were $86 \%$ and $91 \%$ for MRI respectively versus $43 \%$ and $99 \%$ for Mammography.

Morris and co-workers (2003) described a group of 367 high risk women, retrospectively. MRI detected 14 more malignancies than mammography. The study performed by Kriege et al (2004) recruited women with no previous history of breast cancer in several risk groups. The study included 1909 women, age ranging between 19-72 years. Fifty cancers were detected, with a sensitivity of $71 \%$ for MRI, $40 \%$ for XRM (mammography), and $89 \%$ for the combined techniques. MRI specificity was $89.8 \%$ and XRM specificity 95.0\%. Although this study published by Kriege et al (2004) showed a relatively high percentage of breast cancers that were falsenegative on MR images but were diagnosed due only to mammographic abnormalities, this high rate was not observed in the other trials (Kuhl, 2007). Also our study didn't agree with Kriege et al, (2004) there was no false negative on MRI imaging among the malignant cases diagnosed in our study population. Warner et al (2004) performed another study and screened 236 women with gene mutations, including women with a personal history of breast cancer, finding 22 cancers. The sensitivity was $77 \%$ for MRI, $36 \%$ for XRM, and $86 \%$ for both. The specificity was $95.4 \%$ for MRI and $99.8 \%$ for XRM. In a single-centre study conducted by Kuhl et al (2005), 529 women with a life time risk of at least $20 \%$, including women with a personal history of breast cancer, were screened for a mean period of 5 years; 43 cancers were found. Screening included MRI, XRM and ultrasound, with sensitivities of $91 \%, 33 \%$ and $40 \%$, respectively. Combining MRI and XRM gave an overall sensitivity of 93\% for the whole population and $100 \%$ in the group without a personal risk of breast cancer. Specificity of MRI (97.2\%) was equivalent to that of mammography (96.8\%) Mammography alone, and also mammography combined with breast ultrasound, seemed insufficient for early diagnosis of breast cancer in women who are at increased familial risk. They concluded that if MRI is used for surveillance, diagnosis of intraductal and invasive familial or hereditary cancer is achieved with a significantly higher sensitivity and at a more favorable stage. The UK Magnetic Resonance Imaging in Breast Cancer Screening (MARIBS) study, performed by Leach et al (2005), compared MRI with mammography (XRM) in a prospective non-randomised trial in women with at least $50 \%$ risk of carrying gene mutations based on direct testing or family history. MRI was almost twice as sensitive as XRM in this high-risk group (77\% vs $40 \%$ ); 
although the specificity of MRI was lower ( $81 \%$ vs $93 \%$ ). These results show that MRI has considerably greater sensitivity for the detection of breast cancer than XRM in this risk group. Lehman et al (2005) performed a multicenter study, 390 women with a lifetime risk of more than $25 \%$, including previous cancer, received a single screen, detecting six cancers. Sensitivity was not assessed, however, MRI detected all cancers, XRM detected two, and ultrasound one. Another multi-centre study conducted by Sardanelli et al (2007), screened 278 women at high-risk, including previous cancer. Eighteen cancers were detected, giving an MRI sensitivity of $94 \%$, compared with sensitivities of 59\% for XRM, $65 \%$ for ultrasound, and 50\% for clinical breast examination. In a study by Hagen et al (2007) 491 tested mutation carriers received 861 screens. MRI was compared with XRM. Twenty-five cancers were detected, giving a sensitivity of $86 \%$ for MRI and $48 \%$ for XRM. In a single-centre study performed by Riedl et al (2007), 327 women with a high familial risk of breast cancer, including a previous personal history of breast cancer for some, participated in 672 screening rounds. Twenty-eight cancers were detected, with sensitivities of $85.7 \%$ for MRI, $50 \%$ for XRM, and $42.9 \%$ for ultrasound. The specificity was $92 \%$ for MRI and $98 \%$ for XRM. Despite the differences in the study populations and methods used, the studies show the same trends. MRI was invariably a more sensitive technique than XRM, and combining the techniques provided an overall sensitivity of about $90 \%$ (Riedl et al, 2007).
Our results agreed well with these studies mainly as regard the high sensitivity of MRI, however our results shows $100 \%$ sensitivity and higher specificity which is also more than specificity of mammography. This could be attributed to using functional MRI tools, mainly diffusion weighted images which was done in about $60 \%$ of the cases and helped to a great extent in highlighting the pathology among the normal enhancing glandular tissues. None of the above mentioned studies conducted on high risk population utilized such functional tools; they mainly depend on morphological and kinetic assessment of the breast lesions. Yoshikawa et al (2007) performed a study to investigate breast cancerdetecting ability of diffusion weighted magnetic resonance imaging (DW-MRI) by comparing the breast cancer detection rates of DW-MRI and mammography in 48 women who had breast cancer (53 cancer lesions). The breast cancer detection rates by MMG and DW-MRI were $84.9 \%$ and $94.3 \%$, respectively. The mean apparent diffusion coefficient values of IDC (invasive ductal carcinoma) and NIDC (noninvasive ductal carcinoma) were significantly different from that of the normal breast. They concluded that DW-MRI may be useful for detecting breast cancer in a wide age group of women, including young women with dense mammary glands (Yoshikawa et al, 2007). This explains the excellent sensitivity of MRI that we achieved in this study (100\%) and the ability of MRI, including DWI, to detect the lesions which were none visualized in mammography particularly in dense breasts. 


\section{Limitations:}

Although our results are comparable to most of the previous studies, still there are a few limitations. The number of patients included in the study was limited by the relatively short duration (2.5 years) of the study and limited number of patients who undergo breast MR examinations and fit to our inclusion criteria. This is because of cost factor and relative lack of awareness regarding usefulness of MR imaging for the diagnosis of breast cancer. Also the short duration hindered the long term follow up of some of the patients included in our study.

\section{CONCLUSION}

MR imaging of breast is a rapidly evolving modality of excellent sensitivity in detection of breast cancer. The basic drawback of this modality was low specificity for breast malignancy. However, multiple studies including this study have shown that with the improvement in equipment and technique there is gradual increase in specificity.

Generally speaking breast MRI is highly effective in detection and characterization of occult breast lesions in high risk population, with excellent sensitivity and high specificity. This is attributed to the advance in equipment, technique, development and implementation of interpretation guidelines and development of functional MRI tools which contributed to the improving validity of this modality. Also the superiority of MRI compared to mammography, which is more evident in high risk population, supports the use of MRI as an important tool in screening of asymptomatic high risk women.

\section{Case (1):}

42 years old asymptomatic female, with positive family history, mammography showed irregular assymetrical densities in both breasts, US identified a suspicious left retroareolar mass. On CE-MRI the right breast showed an area of non-mass like enhancement in the UOQ with type I benign curve. Left breast showed a prominent retroareolar duct $4.3 \mathrm{~mm}$ with area of abnormal high signal on $\mathrm{T} 2 \mathrm{~W}$ images and post contrast enhancement is seen with diffusion restriction, the $\mathrm{ADC}=0.8$, the time intensity curves of the lesion were type 2 and 3. The lesions were categorized as Left breast lesion; BIRADS 4b, and biopsy was recommended. Right breast lesion; BIRADS III, close follow up was recommended. Histopathology revealed left sided IDC.
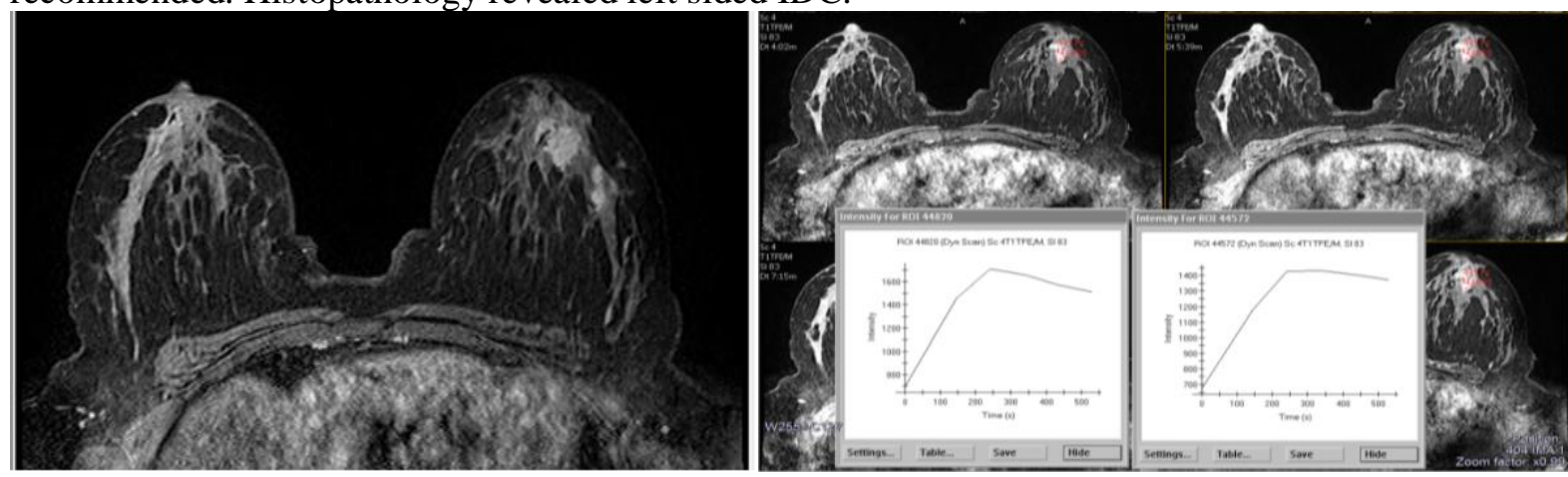

A

B

Fig. 3. A Post-contrast images with fat suppression (T1W TSE post-contrast with SPAIR) showing abnormal high signal and post contrast enhancement in left retroareolar lesion within prominent ducts. B Dynamic multiphase post-contrast study and Time intensity curves. Left breast tetroareolar lesion displaying type $2 \mathrm{~b}$ curve. 


\section{Case (2):}

33 years old female, with positive family history of breast cancer, presented with palpable right retroareolar mass, mammography showed no corresponding architectural distortion or lesions among the dense breast tissues. Contrast enhanced MRI revealed right retroareolar benign looking mass BIRADS II. Follow up was done 6 months later which confirmed benign nature.

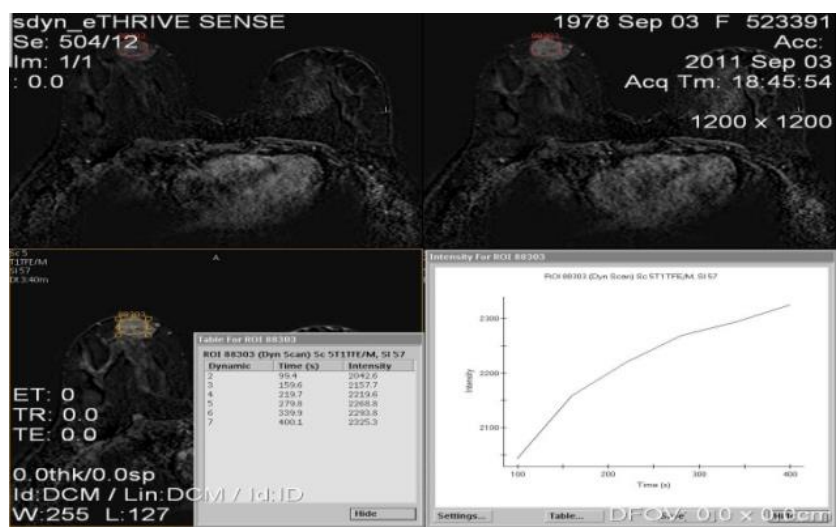

Fig. 4. Dynamic multiphase post-contrast study done in 8 minutes with MIP reconstruction and Time intensity curves. The kinetic data obtained after calculation of the time intensity curve indicates a benign pattern (continuous rising curve with no wash out), type 1 curve.

\section{Case (3):}

32 years old female presented by enlarged left axillary lymph nodes; mammography showed diffuse increased breast density, no definite masses could be identified among the dense breast parenchyma. US showed enlarged left axillary lymph nodes with a query area of architectural distortion in the left breast. CE-MRI revealed bilateral prominent glandular tissue in keeping with pattern of fibroadenosis. The left breast showed a small (about $1 \mathrm{~cm}$ ) rounded retroareolar fibroadenoma. Another rounded rather ill defined lesion was detected showing arterial enhancement in the dynamic phases with type II curve. It showed corresponding diffusion restriction on DW images with ADC value $0.9 \times 10-3$ $\mathrm{mm} / \mathrm{sec}$. This indicates suspicious enhancing lesion in the left breast. The left axilla shows two large rounded lynph nodes with low signal on $\mathrm{T} 1$ and high signal on $\mathrm{T} 2 \mathrm{~W}$ images, with peripheral rim enhancement. Another deeper lymph node was seen with homogenous enhancement of postcontrast T1 weighted images. These showed diffusion restriction on DW images with ADC values $1.18 \times 10$ $3 \mathrm{~mm} / \mathrm{sec} \& 0.6 \times 10-3 \mathrm{~mm} / \mathrm{sec}$ respectively the latter shows rapid upslope and washout. The left breast was categorized as BIRADS IV $\mathrm{b}$ due to the area of mass like architectural distortion with multiple large left axillary adenopathies of suspicous features. Histopathology confirmed malignant nature (Invasive Lobular Carcinoma).
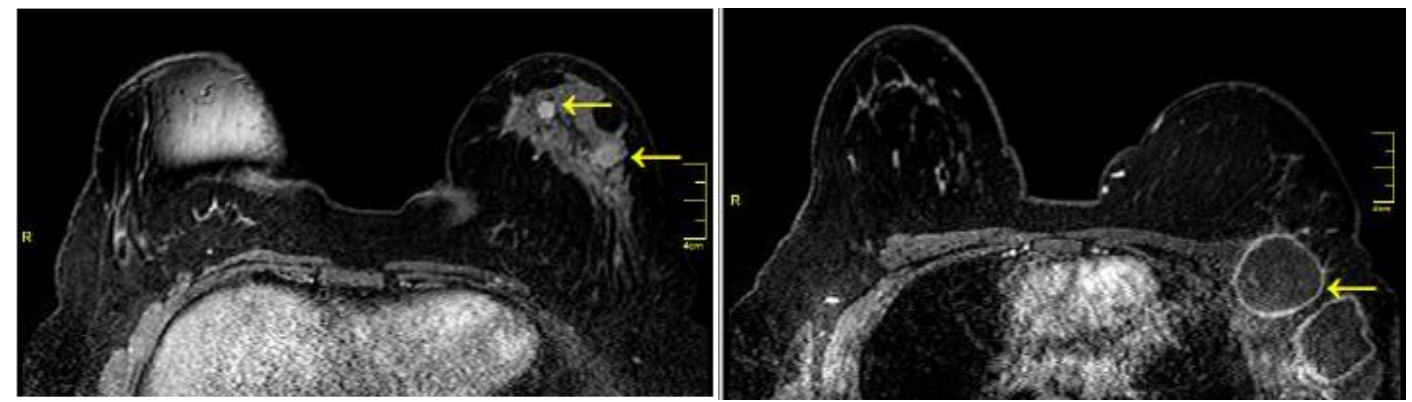

A

B

Fig. 5. Dynamic post-contrast images with fat suppression and MIP reconstruction showing; left breast ill-defined enhanced mass like area at 4 o'clock position, homogenously enhanced retroareolar fibroadenoma (A) and enlarged left axillary lymph nodes displaying rim enhancement (B). 


\section{Case (4):}

58 years old asymptomatic female, with past history of left lumpectomy of a malignant lesion 3 years ago, presented for follow up. Mammography revealed an area of query irregular asymmetric density at right UOQ. CE-MRI revealed an Irregular ill-defined right breast none-mass like lesion at upper outer quadrant. The lesion showed rather speculated margin with radiating striation. It showed significant post contrast enhancement with type III time/intensity curve (consistent with malignant mass), similar adjacent smaller lesions are seen in vicinity (satellites). One large globular lymph node was seen on the right. Right breast none-mass like area of abnormal enhancement was categorized as BIRADS IV with suspicious right axillary lymph node. Histopathology confirmed malignancy IDC.

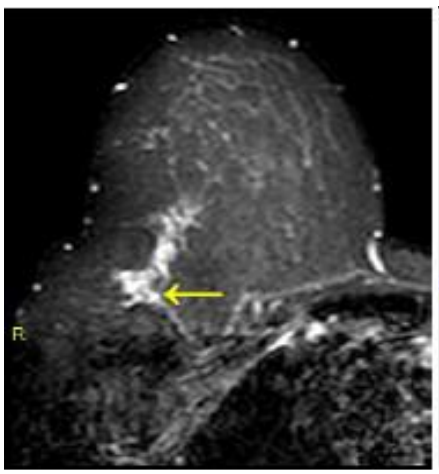

A

B

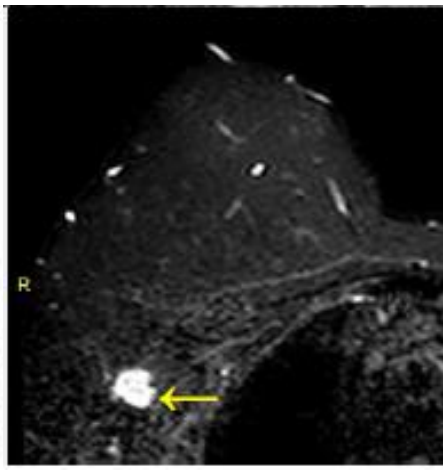

C

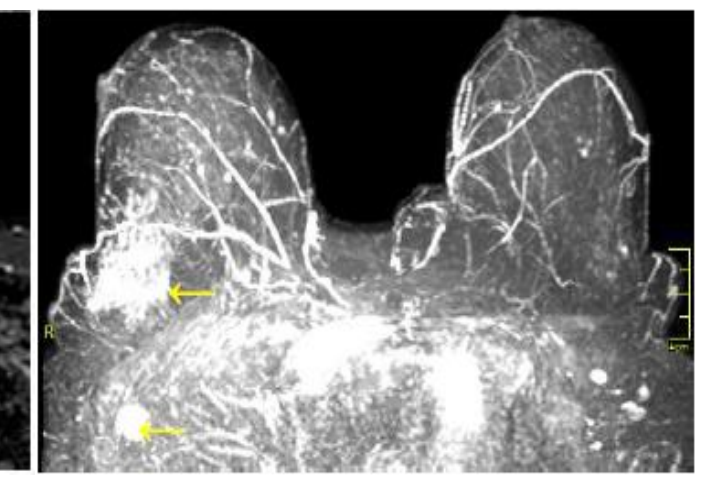
Fig. 6. (A\&B) Dynamic post-contrast images of right breast with fat suppression showing; right UOQ
area of abnormal none-mass like enhancement and enhancing right axillary lymph node. (C) Postcontrast images of right breast with MIP reconstruction showing hypervascular right UOQ ill-defined lesion and right axillary lymph node.

\section{Case (5):}

57 years old female with past history of right UIQ and left UOQ lumpectomies and left axillary evacuation. Left lesion was IDC, while right lesion was benign. Follow up mammography was done and showed right UIQ and left UOQ irregular asymmetric densities. CE-MRI revealed right upper inner and left upper outer quadrant encysted postoperative collection exhibiting bright signal in $\mathrm{T} 2$ \& STIR. The lesions showed marginal enhancement and the kinetic data obtained from the lesion after the dynamic time intensity curves indicates a benign pattern of curves (continuous rise and no wash out) type 1 curves. They were diagnosed as bilateral postoperative seromas, categorized as BIRADS 3. Ultrasound guided aspiration and follow up confirmed benign nature.

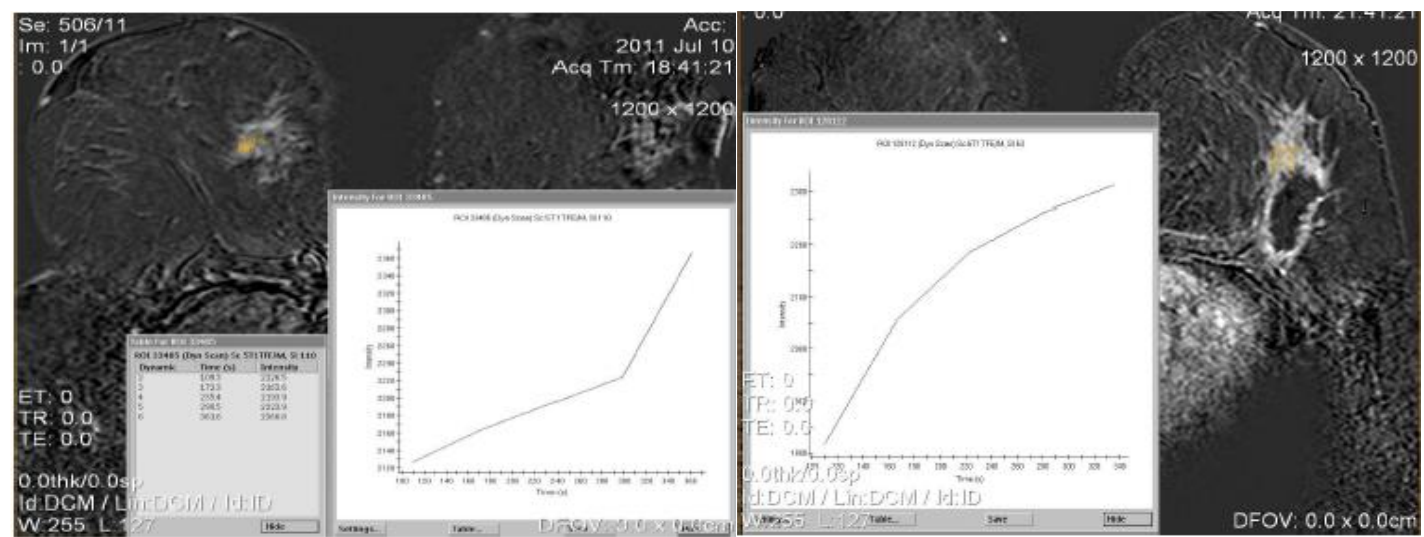

A

B

Fig. 7. (A) Dynamic time intensity curves of the right UIQ lesion marginal enhancement displaying benign type I curves. (B) Dynamic time intensity curves of the left UOQ lesion marginal enhancement displaying benign type I curves. 


\section{References:}

1) Baltzer PAT, Benndorf M, Dietzel M, Gajda M, Runnebaum IB and Kaiser WA. (2010) False-Positive Findings at Contrast-Enhanced Breast MRI: A BI-RADS Descriptor Study. AJR; 194:1658-1663

2) Bedrosian I, Mick R, Orel SG, Schnall M, Reynolds C, Spitz FR, et al. (2003) Changes in the surgical management of patients with breast carcinoma based on preoperative magnetic resonance imaging. Cancer; 98: 46873.

3) Bevers TB. (2008) Chapter 2: Primary prevention of Breast Cancer, screening for early detection of breast cancer, and diagnostic evaluation of clinical and mammographic breast abnormalities. In: Breast Cancer, 2nd edition, edited by Hunt KK, Robb GL, Strom EA, and Ueno NT, Springer; 2:27-55.

4) Bleiweiss IJ and Jaffer S. (2005) Chapter 9: Pathology of Invasive Breast Cancer. In: Breast Cancer, 2nd edition, edited by Roses DF, Elsevier Inc.; 9: 98-110.

5) Fischer U. (2010) MRI of the Breast. In: Clinical MR Imaging: A Practical Approach, 3rd Edition, edited by Reimer P, Parizel PM, Meaney JFM and Stichnoth FA, Springer; 12: 587-610.

6) Friedman PD, Swaminathan S, Herman K and Kalisher L. (2006) Breast MRI: The Importance of Bilateral Imaging. AJR; 187:345-349.

7) Hagen AI, Kvistad KA, Maehle L, Holmen MM, Aase H, Styr B, Vabo A, Apold J, Skaane P, Moller P. (2007) Sensitivity of MRI versus conventional screening in the diagnosis of BRCA-associated breast cancer in a national prospective series. Breast; 16: 367-374.

8) Houssami N, Lord SJ and Ciatto S. (2009) Breast cancer screening: emerging role of new imaging techniques as adjuncts to mammography. MJA; 190: 493-498.

9) Jagannathan NR. (2009) Breast MR. NMR Biomed.; 22: 1-2.

10) Kerlikowske K, Grady D, Barclay J, Sickles EA and Ernster V. (1996) Likelihood ratios for modern screening mammography. Risk of breast cancer based on age and mammographic interpretation. JAMA; 276: 39-43.

11) Kriege M, Brekelmans CT, Boetes C, Besnard PE, et al. (2004) Efficacy of MRI and mammography for breast cancer screening in women with a familial or genetic predisposition. N. Engl. J. Med.; 351: 427437.

12) Kuhl CK, Schmutzler RK, Leutner CC, et al. (2000) Breast MR imaging screening in 192 women proved or suspected to be carriers of a breast cancer susceptibility gene: preliminary results. Radiology; 215:267-79.
13) Kuhl CK. (2007) Current Status of Breast MR Imaging, Part 2. Clinical Applications Radiology; 244:672-691

14) Kuhl CK. (2005) Dynamic Breast Magnetic Resonance Imaging. In: Breast MRI; Diagnosis and Intervention, edited by Morris EA and Liberman L, Springer; 7: 79- 138.

15) Kuhl CK. (2007) The Current Status of Breast MR Imaging: Part I. Choice of Technique, Image Interpretation, Diagnostic Accuracy, and Transfer to Clinical Practice. Radiology; 244:356-378.

16) Leach MO, Boggis CR, Dixon AK, Easton DF, Eeles RA et al. (2005) Screening with magnetic resonance imaging and mammography of a UK population at high familial risk of breast cancer: a prospective multicentre cohort study (MARIBS). Lancet; 365: 1769-1778.

17) Leach MO. (2009) Breast cancer screening in women at high risk using MRI. NMR Biomed.; 22: $17-27$

18) Lehman CD, Blume JD, Weatherall P, Thickman D et al. (2005) Screening women at high risk for breast cancer with mammography and magnetic resonance imaging. Cancer; 103: 1898-1905.

19) Liberman L. (2004) Breast Cancer Screening with MRI - What Are the Data for Patients at High Risk? The new england journal of medicine; 35 : 497- 500

20) Lo G and Cheung PSY.(2008) Use of magnetic resonance imaging for detecting clinically and mammographically occult ductal carcinoma in situ. Hong Kong Med J ; 14:229-232

21) Lord SJ, Lei W, Craft P, Cawson JN, Morris I et al. (2007) A systematic review of the effectiveness of magnetic resonance imaging (MRI) as an addition to mammography and ultrasound in screening young women at high risk of breast cancer. Eur. J. Cancer; 43: 19051917.

22) Macura KJ, Ouwerkerk R et al. (2006) Patterns of Enhancement on Breast MR Images: Interpretation and Imaging Pitfalls. RadioGraphics; 26:1719-1734

23) Morris EA, Liberman L, Ballon DJ et al. (2003) MRI of occult breast carcinoma in a high-risk population. Am J Roentgenol; 181: 619-26

24) Morrow M, Waters J and Morris E. (2011) MRI for breast cancer screening, diagnosis, and treatment. Lancet; 378: 1804-11

25) Mushlin AI, Kouides RW and Shapiro DE. (1998) Estimating the accuracy of screening mammography: a meta-analysis. Am. J. Prev. Med.; 14: 143-153.

26) Odle TG. (2006) Breast MR. Radiologic Technology; 78:45M-65M 
27) Podo F, Sardanelli F, Canese R, et al. (2002) The Italian multi-centre project on evaluation of MRI and other imaging modalities in early detection of breast cancer in subjects at high genetic risk. J Exp Clin Cancer Res; 21(Suppl):115-24.

28) Riedl CC, Ponhold L, Flory D, Weber M et al. (2007) Magnetic resonance imaging of the breast improves detection of invasive cancer, preinvasive cancer, and premalignant lesions during surveillance of women at high risk for breast cancer. Clin Cancer Res.; 13: 6144-6152.

29) Sardanelli F, Podo F, D’Agnolo G, Verdecchia A, et al. (2007) Multicenter comparative multimodality surveillance of women at genetic-familial high risk for breast cancer (HIBCRIT study): interim results. Radiology; 242(3):698-715.

30) Schmitz AC, Veldhuis WB, Stapper G et al. (2008) Contrast-enhanced 3.0-T breast MRI for characterization of breast lesions: increased specificity by using vascular maps. Eur Radiol.; 18: 355-364.

31) Shafqat G, Agha A, Masror I, Rehan M and Afzal S. (2011) Dynamic contrast enhanced MRI breast for lesion detection and characterization with histopathological co relation: Preliminary experience at tertiary care hospital. J Pak Med Assoc; 61:252- 255

32) Spicer DV and Pike MC. (2005) Chapter 5: Risk Factors for Development of Breast Cancer. In: Breast Cancer, 2nd edition, edited by Roses DF, Elsevier Inc.; 5: 4957.

33) Stoutjesdijk MJ, Boetes C, Jager GJ, et al. (2001) Magnetic resonance imaging and mammography in women with a hereditary risk of breast cancer. J Natl Cancer Inst; 93: 1095-1102
34) Tilanus-Linthorst MM, Obdeijn IM, Bartels KC, de Koning HJ, Oudkerk M. (2000) First experiences in screening women at high risk for breast cancer with MR imaging. Breast Cancer Res Treat; 63:53-60.

35) Trecate G, Vergnaghi D, Bergonzi S, et al. (2003) Breast MRI screening in patients with increased familial and/or genetic risk for breast cancer: a preliminary experience. Tumori; 89:125-131.

36) Van de Vijver MJ. (1999) The pathology of familial breast cancer: The preBRCA1/BRCA2era - $\quad$ historical perspectives. Breast Cancer Res; 1:27-30

37) Warner E, Plewes DB, Hill KA, Causer PA, et al. (2004) Surveillance of BRCA1 and BRCA2 mutation carriers with magnetic resonance imaging, ultrasound, mammography, and clinical breast examination. JAMA; 292: 1317-1325.

38) Warner E, Plewes DB, Shumak RS, et al. (2001) Comparison of breast magnetic resonance imaging, mammography, and ultrasound for surveillance of women at high risk for hereditary breast cancer. J Clin Oncol; 19:3524-31.

39) Wiener JI, Schilling KJ, Adami C, and Obuchowski NA. (2005) Assessment of Suspected Breast Cancer by MRI: A Prospective Clinical Trial Using a Combined Kinetic and Morphologic Analysis. AJR; 184:878-886

40) Yoshikawa MI, Ohsumi S, Sugata S, Kataoka M, Takashima S, Kikuchi K and Mochizuki T. (2007) Comparison of breast cancer detection by diffusion weighted magnetic resonance imaging and mammography. Radiat Med; 25:218-223 


\section{توصيف أمراض الثي الغامضة للسكان المعرضين لخطر الإصابة بدرجة كبيرة

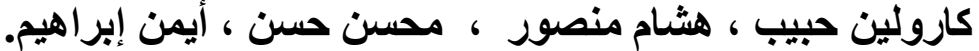

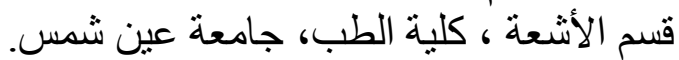

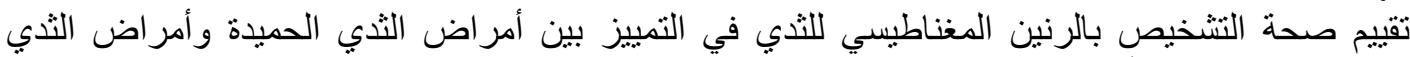

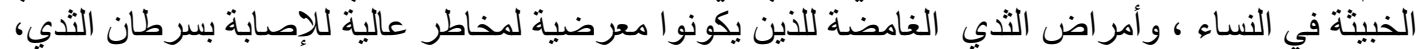
مع النتائج النسيجية و / أو منابعة الاستخدام كمعيار مرجعي.

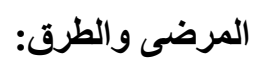

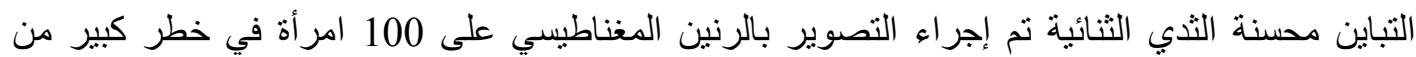

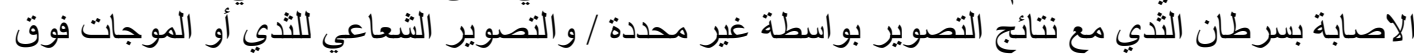

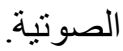

وجرى تقييم الآفات الكثف عنها بو اسطة التصوير بالرنين المغناطيسي التي يمكن أن تمثل الأورام الخبيثة

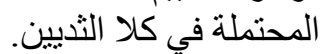

أجريت الثكلية تقييم وتحليل الحركية (تعزيز التباين والوقت / شدة المنحنيات) على كل آفة باستخدام تحليل نتائج العمل المتفاني وبر امج العرض.

و واستخدمت أدوات وظيفية في MR حوالي 60٪ من الحالات للمساعدة في التشخيص التفريقي بين الخبيثة

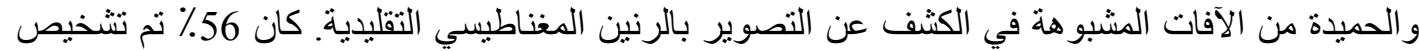

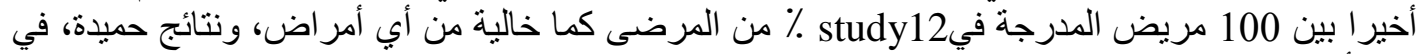
حين أن 31\% من المرضى كانوا الخبيثة

النتائجج:

وكانتDCIS 1). ، IDC24)

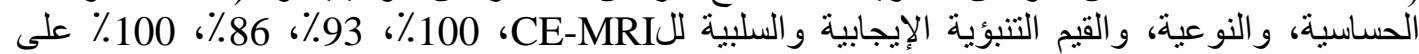

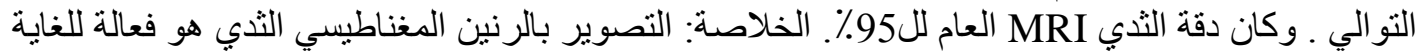

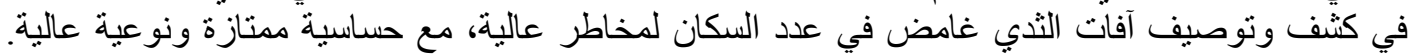
ساهم تطوير أدو ات التصوير بالرنين المغناطيسي الوظيفي لتحسين صحة هذه الطريقة. 